\title{
Combining Ability and Heterosis for Morpho- Physiological Characters on Bread Wheat (Triticum aestivum L.)
}

\author{
Rahul SR* \\ Department of Genetics and Plant Breeding, Rajmata Vijayaraje Scindia Krishi Vishwa Vidhyalaya, India
}

Submission: November 18, 2017; Published: December 20, 2017

${ }^{*}$ Corresponding author: Rahul SR, Department of Genetics and Plant Breeding, Rajmata Vijayaraje Scindia Krishi Vishwa Vidhyalaya, Madhya Pradesh, India, Email: rahulrajpoot146@gmail.com

\begin{abstract}
The present investigation entitled "Combining ability and Heterosis for morpho-physiological characters in bread wheat (Triticum aestivum L.)" was undertaken to study the combining ability and heterosis for grain yield and its components in F1 generation of wheat through Line X Tester analysis. Experiment was conducted with 2 replications and 54 genotypes consisting 10 lines viz., 33rd ESWYT150, 20 ${ }^{\text {th }} H R W Y T 213,20^{\text {th }}$ HRWYT235, 45 ${ }^{\text {th }}$ IBWSN1021, $14^{\text {th }}$ FHBSN6418, 36 $6^{\text {th }}$ SAWSN3065, PBW658, KB2013-03, KB2013, VW921 and 4 testers viz., GW273, GW366, RVW 4106, SUJATA and their 40 crosses made in L X T mating fashion in randomized block design at experimental Research Farm, College of Agriculture, Gwalior, (M.P.) during 2014-2015. Genetic analysis revealed that GCA and SCA variances were significant for all characters, thereby, indicating all traits under the control of both additive and non-additive components inheritance.

The predictability ratios were more than unity, inferring the predominance of additive gene action governing the traits. Good general combiners on the basis of GCA effects were 33rdESWYT150, PBW658, KB2013-03, KB2013 and VW921, GW273 and SUJATA. These parents involved in F2 generations may yield superior trans aggressive recombinant and may exploit either through pedigree selection or progeny selection or mass selection. Segregating generations of good specific combination, 'KB2013-03 × SUJATA', 'PBW658 × SUJATA', 'KB2013 × GW273' and 'VW921 $\times$ SUJATA' consisting good general combining parent would be highly desirable for effective selection. The cross 33rd ESWYT150 $\times$ RVW4106 followed by KB2013-03 $\times$ SUJATA and KB2013-03 $\times$ RVW4106 was recognized as best heterotic cross as well as high per se performance for grain yield and it exhibited highly significant positive heterosis over both the better and mid parent.
\end{abstract}

Keywords: Combining ability; GCA; SCA; Relative heterosis; Heterobeltiosis

\section{Introduction}

Wheat is the principal food crop in most areas of the world and also occupies prominent position in Indian agriculture after rice. It is nutritionally important cereal essential for the food security, poverty alleviation and for livelihoods. It is widely cultivated as staple food crop among the cereals and is contributing about $30 \%$ to the food basket of the country. India is the second largest producer of wheat in the world with the production around 75 million tonnes during the last decade and it is a major contributor to the food security system in India, occupying nearly 30.37 million hectares, producing 90.78 million tonnes and productivity 29.89q/ha and in Madhya Pradesh, grown in 5.56 million hectares with production of 13.37 million tonnes and productivity of 24.05q/ha (Anonymous 2014-2015). The substantial improvement in production is utmost necessary not only to meet ever increasing food requirement for domestic consumption, but also for export to earn foreign exchange. To feed the growing population, the country's wheat requirement by 2030 has been estimated at 100 million metric tonnes and to achieve this target, wheat production has to be increased at the rate of $<1$ per annum and this can be achieved through horizontal approach i.e. by increasing area under cultivation or through vertical approach i.e. varietal/hybrid improvement, which is one of the strongest tool to take a quantum jump in production and productivity under various agro- climatic conditions.

Triticum aestivum (bread wheat) $(2 n=6 x=42)$ is an allohexaploid produced from two separate naturally occurring hybridization events. The initial hybridization, that occurred between the two grass species Triticum urartu $(2 n=2 x=14)$ (the A genome donor), and Triticum speltoides $(2 n=2 x=14)$ (B genome donor). This new species would have been tetraploid wheat $(2 \mathrm{n}=4 \mathrm{x}=28) \quad$ viz., Triticum turgidum var. durum (Durum wheat). Hexaploid wheat arose as a result of a second hybridization between the new tetraploid and a third diploid species $(2 \mathrm{n}=2 \mathrm{x}=14)$ viz., Triticum tauschii (D genome donor). 
Again, doubling chromosome must have occurred in order to produce a fertile individual. This new species would then have 42 chromosomes; i.e. six complete genomes each of 7 chromosomes.

\section{Materials and Methods}

The experimental material consisted 54 genotypes consisting 10 lines viz., 33 ${ }^{\text {rd }}$ ESWYT150, 20 ${ }^{\text {th }} H R W Y T 213,20^{\text {th }} H R W Y T 235$, $45^{\text {th }}$ IBWSN1021, 14 ${ }^{\text {th }}$ FHBSN6418, 36 ${ }^{\text {th }}$ SAWSN3065, PBW658, KB2013-03, KB2013, VW921 and 4 testers viz., GW273, GW366, RVW 4106, SUJATA and their 40 crosses made in L X T mating fashion in randomized block design at experimental Research Farm, College of Agriculture, Gwalior, (M.P.) during 2014-2015. Observations were recorded on randomly selected 5 tagged plants for grain yield per plant and different yield contributing traits viz., days to heading, days to maturity, plant height, tillers per plant, spike length, 1st internodes length, weight of spikes per plant, weight of grains per spike, grains per spike, test weight, canopy temperature index, biological yield and harvest index. The analysis was carried out by adopting Kempthorne 0 [1] for estimation of variances components and combining ability effects. Heterosis over mid parents and better parents were determined as per standard procedure. Significance of heterosis value was tested using ' $t$ ' test.

\section{Results and Discussion}

Line $\times$ tester analysis techniques has been extensively used in almost all the major field crops to estimates GCA and SCA variances and effect and to understand In the present research work, variances due to both lines and testers were significant for days to heading, plant height, tillers per plant, spike length, weight of spikes per plant, grains per spike, test weight, grain yield per plant, canopy temperature index, biological yield and harvest index. However, lines were significant for days to maturity, 1st inter-node length, weight of grains per spike. Variances due to Line $\times$ tester were significant for all the 14 characters [2,3] also reported similar results). In the present study both GCA and SCA variances were significant for most of the characters, thereby, indicating importance of both additive as well as non additive components of genetic variance in the control of these traits (Table 1). Singh et al. [2] \& Kumar et al. [4] also reported similar results.

Table 1: Analysis of variance for combining ability for Grains yield and yield contributing characters in wheat.

\begin{tabular}{|c|c|c|c|c|c|c|c|}
\hline Sources & DF & $\begin{array}{c}\text { Days to } \\
\text { Heading }\end{array}$ & $\begin{array}{c}\text { Days to } \\
\text { Maturity }\end{array}$ & Tillers/Plant & $\begin{array}{l}1^{\text {st }} \text { Inter-Node } \\
\text { Length }\end{array}$ & Spike Length & $\begin{array}{c}\text { Wt. of Spike/ } \\
\text { Plant }\end{array}$ \\
\hline Lines & 9 & $2.48^{* *}$ & $4.47^{* *}$ & $0.91^{* *}$ & $0.09^{* *}$ & $0.76^{* *}$ & $6.10^{* *}$ \\
\hline Tester & 3 & $3.90^{* *}$ & 0.55 & 0.27 & 0.01 & $0.90^{* *}$ & $0.54^{*}$ \\
\hline $\mathrm{LXT}$ & 27 & $2.14^{* *}$ & $5.49^{* *}$ & $0.38^{* *}$ & $0.08^{* *}$ & $2.41^{* *}$ & $3.58^{* *}$ \\
\hline Error & 53 & 0.4 & 0.49 & 0.08 & 0.01 & 0.01 & 0.17 \\
\hline
\end{tabular}

\begin{tabular}{|c|c|c|c|c|c|c|c|c|}
\hline Sources & DF & $\begin{array}{c}\text { Grains } \\
\text { Weight/ } \\
\text { Spike }\end{array}$ & Grains/Spike & Test Weight & $\begin{array}{c}\text { Grains } \\
\text { Yield/Plant }\end{array}$ & $\begin{array}{c}\text { Canopy } \\
\text { Temperature } \\
\text { Index }\end{array}$ & $\begin{array}{c}\text { Biological } \\
\text { Yield }\end{array}$ & $\begin{array}{c}\text { Harvest } \\
\text { Index }\end{array}$ \\
\hline Lines & 9 & $0.08^{* *}$ & $16.35^{* *}$ & $48.25^{* *}$ & $7.40^{* *}$ & $0.0011^{* *}$ & $8.66^{* *}$ & $64.79^{* *}$ \\
\hline Tester & 3 & 0.01 & $26.96^{* *}$ & $2.42^{* *}$ & $0.42^{* *}$ & $0.0004^{*}$ & $2.32^{* *}$ & $3.69^{* *}$ \\
\hline L X T & 27 & $0.03^{* *}$ & $79.12^{* *}$ & $14.63^{* *}$ & $2.29^{* *}$ & $0.0010^{* *}$ & $3.45^{* *}$ & $15.93^{* *}$ \\
\hline Error & 53 & 0.01 & 1.06 & 0.18 & 0.02 & 0.0001 & 0.32 & 1.57 \\
\hline
\end{tabular}

\section{Genetic components}

Genetic component of variances is assessed through the estimates of GCA and SCA variances. The additive genetic variance is equal to GCA variance and dominance variance is equal to SCA variance. In the present research, the genetic component's magnitudes showed that the additive components of variances were higher compared to dominance components of variances for all characters. The ratio of genetic components " $\sigma 2 \mathrm{gca} /$ б2sca" also showed more than one, indicating predominance of additive variances for almost all characters (Table 2). Present finding are in confirmation with Kandil et al. [5], Khiabani et al. [6] \& Samier \& Ismail (2015) were recorded predominance of additive variances for all the traits.

Table 2: Genetic components for Grains yield and its attributes in wheat.

\begin{tabular}{|c|c|c|c|c|c|c|c|}
\hline & $\begin{array}{l}\text { Days to } \\
\text { Heading }\end{array}$ & $\begin{array}{l}\text { Days to } \\
\text { Maturity }\end{array}$ & Plant Height & Tillers/Plant & $\begin{array}{l}1^{\text {st }} \text { Inter-Node } \\
\text { Length }\end{array}$ & Spike Length & $\begin{array}{c}\text { Wt. Of Spike/ } \\
\text { Plant }\end{array}$ \\
\hline \multicolumn{8}{|c|}{ Covariances } \\
\hline Cov HS (Line) & 0.04 & -0.05 & -2.77 & 0.03 & 0 & -0.08 & 0.13 \\
\hline Cov HS (Tester) & 0.09 & -0.62 & -9.06 & -0.01 & -0.01 & -0.19 & -0.38 \\
\hline $\begin{array}{c}\text { Cov HS } \\
\text { (Average) }\end{array}$ & 0.01 & -0.02 & -0.45 & 0 & 0 & -0.01 & 0.01 \\
\hline
\end{tabular}


Agricultural Research \& Technology: Open Access Journal

\begin{tabular}{|c|c|c|c|c|c|c|c|c|}
\hline Cov FS & 5.85 & 4.9 & 45.96 & 1.06 & 0.1 & 2.07 & 6.85 \\
\hline \multicolumn{7}{|c|}{ Genetic Components } \\
\hline$\sigma 2$ GCA (Lines) & 0.01 & -0.02 & -0.45 & 0 & 0 & -0.01 & 0.01 \\
\hline$\sigma 2 \mathrm{GCA}$ (Testers) & 0.02 & -0.06 & -1.81 & 0.01 & 0 & -0.05 & 0.03 \\
\hline $\begin{array}{c}\sigma 2 \mathrm{GCA} \\
\text { (Parents) }\end{array}$ & 11.77 & -33.1 & -526.51 & -0.16 & -0.5 & -11.43 & -17.24 \\
\hline$\sigma 2 \mathrm{SCA}$ & 0.87 & 2.5 & 39.93 & 0.15 & 0.04 & 1.2 & 1.7 \\
\hline$\sigma 2 \mathrm{GCA} / \sigma 2 \mathrm{SCA}$ & 13.52 & -13.26 & -13.19 & -1.06 & -12.87 & -9.54 & -10.11 \\
\hline$\sigma 2 \mathrm{~A}$ & 23.54 & -66.19 & -1053.02 & -0.31 & -1 & -22.87 & -34.48 \\
\hline$\sigma 2 \mathrm{D}$ & 0.87 & 2.5 & 39.93 & 0.15 & 0.04 & 1.2 & 1.7 \\
\hline
\end{tabular}

\begin{tabular}{|c|c|c|c|c|c|c|c|}
\hline & $\begin{array}{c}\text { Grains } \\
\text { Weight/Spike }\end{array}$ & Grains/Spike & Test Weight & $\begin{array}{l}\text { Grains Yield/ } \\
\text { Plant }\end{array}$ & $\begin{array}{c}\text { Canopy } \\
\text { Temperature } \\
\text { Index }\end{array}$ & $\begin{array}{l}\text { Biological } \\
\text { Yield }\end{array}$ & Harvest Index \\
\hline \multicolumn{8}{|c|}{ Covariances } \\
\hline Cov HS (Line) & 0 & -3.14 & 1.68 & 0.26 & 0 & 0.65 & 6.11 \\
\hline Cov HS (Tester) & 0 & -6.52 & -1.53 & -0.23 & -0.0001 & -0.06 & -0.61 \\
\hline $\begin{array}{c}\text { Cov HS } \\
\text { (Average) }\end{array}$ & 0 & -0.46 & 0.17 & 0.03 & 0 & 0.03 & 0.25 \\
\hline Cov FS & 0.08 & 55.12 & 52.38 & 8.1 & 0.0014 & 10.79 & 67.04 \\
\hline \multicolumn{8}{|c|}{ Genetic Components } \\
\hline$\sigma 2 \mathrm{GCA}$ (Lines) & 0 & -0.46 & 0.17 & 0.03 & 0 & 0.03 & 0.25 \\
\hline$\sigma 2 \mathrm{GCA}($ Testers) & 0 & -1.82 & 0.67 & 0.1 & 0 & 0.11 & 1.02 \\
\hline $\begin{array}{c}\sigma 2 \mathrm{GCA} \\
\text { (Parents) }\end{array}$ & -0.09 & -401.83 & -45.77 & -7.03 & 0 & -2.09 & -30.72 \\
\hline$\sigma 2 \mathrm{SCA}$ & 0.01 & 39.03 & 7.23 & 1.13 & 0 & 1.56 & 7.18 \\
\hline$\sigma 2 \mathrm{GCA} / \sigma 2 \mathrm{SCA}$ & -6.58 & -10.29 & -6.33 & -6.2 & -7.8 & -1.34 & -4.28 \\
\hline$\sigma 2 \mathrm{~A}$ & -0.18 & -803.67 & -91.54 & -14.05 & -0.01 & -4.19 & -61.45 \\
\hline$\sigma 2 \mathrm{D}$ & 0.01 & 39.03 & 7.23 & 1.13 & 0 & 1.56 & 7.18 \\
\hline
\end{tabular}

\section{Combining ability}

Combining ability plays major role in the evaluation of inbred in terms of their breeding values; and this will help to decide efficient breeding method to be applied in segregating generation.

\section{General combining ability effects}

It is primarily a function of additive genetic variance; it helps in the selection of suitable good general combining parents for hybridization. Data presented in Table 3 revealed that 5 lines viz., 33rd ESWYT150, PBW 658, KB 2013-03, KB 2013 and VW921 reported significant and positive GCA effect for grain yield, test weight, weight of spike/plant, biological yield and harvest index, thereby, suggesting good general combiner for these traits. Tester 'GW273' was good general combiner for grain yield, test weight and biological yield, whereas, tester SUJATA registered significant and positive GCA effect for grain yield, days to heading, spike length and harvest index, thereby, suggested good general combiner for these traits.

Table 3: Parents showing significant GCA effects for grain yield along with GCA effects for other attributes.

\begin{tabular}{|c|c|c|c|c|c|c|c|c|c|c|c|}
\hline \multirow{2}{*}{ Parents } & $\begin{array}{c}\text { Lines/ } \\
\text { Tester }\end{array}$ & \multicolumn{2}{|c|}{$\begin{array}{c}\text { Grain Yield/ } \\
\text { Plant(G) }\end{array}$} & \multicolumn{2}{|c|}{ Days to Heading } & \multicolumn{2}{c|}{ Days to Maturity } & \multicolumn{2}{c|}{ Plant Height(Cm) } & \multicolumn{2}{c|}{ Tillers/Plant (Cm) } \\
\hline & Mean & GCA & Mean & GCA & Mean & GCA & Mean & GCA & Mean & GCA \\
\hline GW 273 & T & 17.91 & $0.17^{*}$ & 85.5 & 0.1 & 135 & 0.22 & 104.8 & -0.05 & 8.3 & 0.08 \\
\hline SUJATA & T & 17.33 & $0.07^{*}$ & 85.5 & $0.55^{* *}$ & 137 & -0.09 & 104.6 & -0.09 & 8.3 & -0.12 \\
\hline VW 921 & L & 16.56 & $0.75^{* *}$ & 87.5 & $0.45^{* *}$ & 135 & $-0.60^{* *}$ & 103.9 & $2.83^{* *}$ & 7.1 & -0.01 \\
\hline PBW 658 & L & 16.46 & $0.47^{* *}$ & 86.5 & $-1.05^{* *}$ & 138.5 & -0.47 & 104.7 & $-1.95^{* *}$ & 7 & $-0.21^{* *}$ \\
\hline $\begin{array}{c}33^{\text {rd }} \\
\text { ESWT } \\
150\end{array}$ & L & 16.23 & $1.51^{* *}$ & 87 & -0.05 & 139 & -0.35 & 100.2 & $-1.85^{* *}$ & 7.2 & $0.77^{* *}$ \\
\hline
\end{tabular}


Agricultural Research \& Technology: Open Access Journal

\begin{tabular}{|c|c|c|c|c|c|c|c|c|c|c|c|}
\hline $\begin{array}{c}\text { KB 2013- } \\
03\end{array}$ & $\mathrm{~L}$ & 16.11 & $0.72^{* *}$ & 89.5 & -0.05 & 136.5 & -0.47 & 109.6 & $1.75^{* *}$ & 6.9 & 0.04 \\
\hline KB 2013 & $\mathrm{~L}$ & 14.98 & $0.68^{* *}$ & 88 & 0.08 & 137 & $-0.60^{* *}$ & 109.6 & 0.45 & 6.9 & $-0.51^{* *}$ \\
\hline \multirow[t]{2}{*}{ Parents } & $\begin{array}{l}\text { Lines/ } \\
\text { Tester }\end{array}$ & \multicolumn{2}{|c|}{$\begin{array}{l}\text { 1stinter-Node } \\
\text { Length }(\mathrm{Cm})\end{array}$} & \multicolumn{2}{|c|}{ Spike Length(Cm) } & \multicolumn{2}{|c|}{$\begin{array}{l}\text { Spike Weight/ } \\
\text { Plant(G) }\end{array}$} & \multicolumn{2}{|c|}{$\begin{array}{l}\text { Grain Weight/ } \\
\text { Spike(G) }\end{array}$} & \multicolumn{2}{|c|}{ Grains /Spike } \\
\hline & & Mean & GCA & Mean & GCA & Mean & GCA & Mean & GCA & mean & GCA \\
\hline GW 273 & $\mathrm{~T}$ & 3.11 & $0.17^{*}$ & 10.75 & $-0.17^{* *}$ & 24.46 & 0.16 & 2.26 & 0 & 68.1 & -1.09 \\
\hline SUJATA & $\mathrm{T}$ & 2.68 & $0.07^{*}$ & 11.32 & $0.28^{* *}$ & 23.78 & 0.12 & 2.11 & 0.02 & 70.8 & 1.52 \\
\hline VW 921 & L & 2.77 & $0.75^{* *}$ & 11.11 & 0.05 & 23.08 & $0.66^{* *}$ & 2.04 & 0.06 & 66.5 & -0.76 \\
\hline PBW 658 & $\mathrm{~L}$ & 2.9 & $0.47^{* *}$ & 10.29 & $-0.15^{* *}$ & 23.41 & $0.32^{* *}$ & 2.12 & 0.04 & 60.8 & 0.54 \\
\hline $\begin{array}{c}33^{\text {rd }} \\
\text { ESWYT } \\
150\end{array}$ & $\mathrm{~L}$ & 3.02 & $1.51^{* *}$ & 10.43 & $-0.61^{* *}$ & 21.22 & $1.36^{* *}$ & 2 & $0.13^{* *}$ & 61.9 & $-2.11^{* *}$ \\
\hline $\begin{array}{c}\text { KB 2013- } \\
03\end{array}$ & $\mathrm{~L}$ & 3.02 & $0.72^{* *}$ & 8.94 & $-0.23^{* *}$ & 21.01 & $0.68^{* *}$ & 2.07 & $0.07^{* *}$ & 54.2 & $0.94^{* *}$ \\
\hline KB 2013 & $\mathrm{~L}$ & 2.91 & $0.68^{* *}$ & 11.03 & $0.22^{* *}$ & 20.09 & $0.58^{* *}$ & 2.02 & $0.07^{* *}$ & 67.3 & -0.36 \\
\hline
\end{tabular}

\begin{tabular}{|c|c|c|c|c|c|c|c|c|c|}
\hline \multirow{2}{*}{ Parents } & $\begin{array}{c}\text { Lines/ } \\
\text { Tester }\end{array}$ & \multicolumn{2}{|c|}{ Test Weight(G) } & \multicolumn{2}{c|}{$\begin{array}{c}\text { Canopy Temperature } \\
\text { Index }\end{array}$} & \multicolumn{2}{c|}{ Biological Yield(G) } & \multicolumn{2}{c|}{ Harvest Index (\%) } \\
\hline & & Mean & GCA & Mean & GCA & Mean & GCA & Mean & GCA \\
\hline GW 273 & T & 44.78 & $0.42^{* *}$ & 0.41 & $-0.01^{* *}$ & 32.7 & $0.43^{* *}$ & 54.75 & 0.11 \\
\hline SUJATA & T & 43.33 & 0.13 & 0.36 & 0 & 33.48 & -0.15 & 51.75 & $0.56^{*}$ \\
\hline VW 921 & L & 41.54 & $1.99^{* *}$ & 0.36 & $0.02^{* *}$ & 35.04 & $-0.73^{* *}$ & 47.27 & $2.79^{* *}$ \\
\hline PBW 658 & L & 41.82 & $1.11^{* *}$ & 0.35 & $-0.01^{* *}$ & 35.76 & $0.96^{* *}$ & 46.59 & $2.36^{* *}$ \\
\hline $\begin{array}{c}33^{\text {rd }} \text { ESWYT } \\
\text { 150 }\end{array}$ & L & 40.79 & $3.73^{* *}$ & 0.36 & -0.01 & 39.4 & $2.18^{* *}$ & 41.22 & $1.57^{* *}$ \\
\hline KB 2013-03 & L & 40.49 & $2.02^{* *}$ & 0.36 & 0.01 & 35.29 & $1.05^{* *}$ & 45.65 & $3.28^{* *}$ \\
\hline KB 2013 & L & 38.69 & $1.64^{* *}$ & 0.37 & $0.01^{* *}$ & 37.96 & $-0.92^{* *}$ & 43.09 & $3.00^{* *}$ \\
\hline
\end{tabular}

Lines 33rd ESWYT150 was also reported significant and positive GCA effect for tillers/plant and weight of grains/ plant. Line KB2013-03 also exhibited significant positive GCA effect for plant height, weight of grain/spike and grains/spike. Significant positive GCA effect was reported in KB2013 and VW921 for canopy temperature index. These genotypes can be used in the development of high yielding varieties through the pedigree selection and progeny selection or mass selection in later generations in promising segregating generations in wheat Present finding are in confirmation with Kandil et al. [5], Samier and Ismail (2015), Kalhoro et al. [7], Aslam et al. [8], Singh et al. [2], Raj \& Kandalkar [9], Kumar et al. [4] \& Kapoor et al. [3].

\section{Specific combining ability Effects}

It is mainly a function of dominance variances; it helps in the identification of superior cross combination for commercial exploitation of heterosis. In self-pollinated crops like wheat, SCA effects are not much important as they are mostly related to non-additive gene effects excluding those of arising from complementary gene action or linkage effects they cannot be fixed in pure lines. Further superiority of the hybrids might not indicate their ability to yield transgressive segregates; rather SCA would provide satisfactory criteria. However, if a cross combination exhibiting high SCA as well as high per se performance having at least one parent as good general combiner for a specific trait, it is expected to throw desirable transgressive segregants in later generations. Data presented in Table 4 revealed that 19 crosses showed significant and positive SCA effect, thereby, indicating good specific combinations for grain yield and other attributing traits. Crosses viz., KB2013-03×SUJATA', KB2013×GW273', PBW658 $\times$ SUJATA' and 'VW921×SUJATA' registered best good specific combiner for grain/yield because these crosses were the results of good $\mathrm{x}$ good general combiners and reported significant positive SCA effect. Crosses '33rd ESWYT150×GW366', ' $33^{\text {rd }}$ ESWYT150 $\times$ RVW4106', '20 $0^{\text {th }}$ HRWYT213×GW273', ' $20^{\text {th }} \quad$ HRWYT213 $\times$ SUJATA', ' $20^{\text {th }} \quad H R W Y T 235 \times$ SUJATA', ' $45^{\text {th }}$ IBWSN1021×GW273', '14 $4^{\text {th }}$ FHBSN6418 $\times$ SUJATA', 'KB201303×GW366', 'KB2013×RVW4106' and 'VW921×GW366' were the result of good $\mathrm{x}$ poor general combiner and also reported significant positive SCA effect for grain yield. Rest of the crosses viz., ' $45^{\text {th }}$ IBWSN1021 × RVW4106', ' $36^{\text {th }}$ SAWSN3065 × RVW4106', ' $36^{\text {th }}$ SAWSN3065 $\times$ GW366', ' $14^{\text {th }}$ FHBSN6418 $\times$ GW366', ' $45^{\text {th }}$ IBWSN1021 × RVW4106', '20 $0^{\text {th }}$ HRWYT235 × GW366' were result of poor $x$ poor general combiner but exhibited significant positive SCA effect for grain/yield, thereby, suggesting good specific combiner for these traits. Present finding are in confirmation with Kandil et al. [5], Kalhoro et al. [7], Aslam et al. [8], Singh et al. [2], Raj \& Kandalkar [9], Kumar et al. [4], Kapoor et al. [3]. 
Table 4: Crosses showing significant SCA effects for grain yield and its attributes.

\begin{tabular}{|c|c|c|c|c|c|c|c|c|}
\hline \multirow[t]{2}{*}{ SN } & \multirow[t]{2}{*}{ Crosses } & \multicolumn{2}{|c|}{ Grain Yield/Plant(G) } & \multirow{2}{*}{$\begin{array}{c}\begin{array}{c}\text { Days to } \\
\text { Heading }\end{array} \\
\text { SCA }\end{array}$} & \multirow{2}{*}{$\begin{array}{c}\begin{array}{c}\text { Days to } \\
\text { Maturity }\end{array} \\
\text { SCA }\end{array}$} & \multirow{2}{*}{$\begin{array}{c}\begin{array}{c}\text { Plant } \\
\text { Height(Cm) }\end{array} \\
\text { SCA }\end{array}$} & \multirow{2}{*}{$\begin{array}{c}\text { Tillers/ } \\
\text { Plant }\end{array}$} & \multirow{2}{*}{$\begin{array}{c}\begin{array}{c}1^{\text {st }} \text { Inter- } \\
\text { Node } \\
\text { Length }(\mathrm{Cm})\end{array} \\
\text { SCA }\end{array}$} \\
\hline & & Mean & SCA & & & & & \\
\hline 1 & $33^{\text {rd }}$ ESWYT $150 \times$ GW 366 & 17.87 & $0.59 * *$ & -0.5 & $-1.45^{* *}$ & $2.73^{* *}$ & 0.36 & $0.16^{* *}$ \\
\hline 2 & $33^{\text {rd }}$ ESWYT $150 \times$ RVW 4106 & 18.06 & $0.72^{* *}$ & 0.15 & $-1.10^{* *}$ & $5.99 * *$ & $0.56^{* *}$ & $0.14^{* *}$ \\
\hline 3 & $20^{\text {th }}$ HRWYT $213 \times$ GW 273 & 16.89 & $1.67^{* *}$ & -0.1 & -0.1 & $1.85^{* *}$ & $0.59^{* *}$ & $-0.26^{* *}$ \\
\hline 4 & $20^{\text {th }}$ HRWYT $213 \times$ SUJATA & 15.44 & $0.23^{* *}$ & -0.05 & -0.85 & $5.59 * *$ & 0.19 & 0.09 \\
\hline 5 & $20^{\text {th }}$ HRWYT $235 \times$ GW 366 & 15.57 & $1.27^{* *}$ & -0.5 & $-1.70^{* *}$ & 0.83 & -0.16 & $-0.20^{* *}$ \\
\hline 6 & $20^{\text {th }}$ HRWYT $235 \times$ SUJATA & 14.8 & $0.29^{* *}$ & -0.05 & 0.15 & $1.62^{* *}$ & 0.17 & 0.13 \\
\hline 7 & $45^{\text {th }}$ IBWSN $1021 \times$ GW 273 & 15.7 & $0.67^{* *}$ & $2.52^{* *}$ & -0.1 & $6.75^{* *}$ & -0.31 & 0.24 \\
\hline 8 & $45^{\text {th }}$ IBWSN $1021 \times$ RVW 4106 & 15.87 & $1.09 * *$ & $-1.23^{* *}$ & $1.35^{* *}$ & $1.86^{* *}$ & -0.24 & $-0.31^{* *}$ \\
\hline 9 & $14^{\text {th }}$ FHBSN6 $4188 \times \mathrm{GW} \times 366$ & 15.79 & $0.54^{* *}$ & -0.63 & 0.92 & $2.98^{* *}$ & 0.24 & $0.18^{* *}$ \\
\hline 10 & $14^{\text {th }}$ FHBSN6 $4188 \times$ SUJATA & 15.87 & $0.41^{* *}$ & 0.8 & $-1.72^{* *}$ & -0.03 & -0.23 & $-0.24^{* *}$ \\
\hline 11 & $36^{\text {th }}$ SAWSN $3065 \times$ GW 366 & 16.31 & $0.82^{* *}$ & 0.63 & $-1.33^{* *}$ & 0.15 & 0.14 & 0.05 \\
\hline 12 & $36^{\text {th }}$ SAWSN $3065 \times$ RVW 4106 & 16.06 & $0.51^{* *}$ & -0.73 & -0.47 & $4.41^{* *}$ & 0.16 & 0 \\
\hline 13 & PBW $658 \times$ SUJATA & 16.89 & $0.42^{* *}$ & -0.55 & 0.03 & $7.62^{* *}$ & 0.37 & $0.12^{*}$ \\
\hline 14 & KB 2013-03 ×GW366 & 17.16 & $0.66^{* *}$ & 0 & -0.83 & $4.63^{* *}$ & -0.81 & $-0.17^{* *}$ \\
\hline 15 & KB 2013-03 × SUJATA & 18 & $1.29^{* *}$ & 0.95 & $0.97^{*}$ & $2.32^{* *}$ & $-0.48^{* *}$ & $0.21^{* *}$ \\
\hline 16 & KB $2013 \times$ GW 273 & 17.27 & $0.49^{* *}$ & -0.73 & $-1.10^{* *}$ & $5.47^{* *}$ & $-0.63^{* *}$ & $0.17^{* *}$ \\
\hline 17 & KB $2013 \times$ RVW 4106 & 17.42 & $0.90^{* *}$ & -0.48 & -0.85 & 0.79 & -0.27 & $-0.18^{* *}$ \\
\hline 18 & VW $921 \times$ RVW 4106 & 17.43 & $0.91^{* *}$ & 0 & $1.30^{* *}$ & $3.75^{* *}$ & 0.04 & 0.13 \\
\hline 19 & VW $921 \times$ SUJATA & 17.01 & $0.27^{* *}$ & 0.45 & -0.85 & $-2.46^{* *}$ & -0.03 & $-0.20^{* *}$ \\
\hline
\end{tabular}

\begin{tabular}{|c|c|c|c|c|c|c|}
\hline SN & Crosses & $\begin{array}{c}\text { Spike } \\
\text { Length }(\mathrm{Cm})\end{array}$ & $\begin{array}{l}\text { Spikes Weight/ } \\
\text { Plant(G) }\end{array}$ & $\begin{array}{c}\text { Grain } \\
\text { Weight/ } \\
\text { Spike(G) }\end{array}$ & $\begin{array}{l}\text { Grains/ } \\
\text { Spike }\end{array}$ & $\begin{array}{c}\text { Test } \\
\text { Weight(G) }\end{array}$ \\
\hline & & SCA & SCA & SCA & SCA & SCA \\
\hline 1 & $33^{\text {rd }}$ ESWYT $150 \times$ GW 366 & $0.22^{* *}$ & $0.84^{* *}$ & $0.11^{*}$ & $-1.50^{* *}$ & $1.56^{* *}$ \\
\hline 2 & 33 $3^{\text {rd }}$ ESWYT $150 \times$ RVW 4106 & $-0.70^{* *}$ & $1.24^{* *}$ & -0.01 & $-3.56^{* *}$ & $1.65^{* *}$ \\
\hline 3 & $20^{\text {th }}$ HRWYT $213 \times$ GW 273 & $0.27^{* *}$ & $1.96^{* *}$ & 0.08 & 0.71 & $4.25^{* *}$ \\
\hline 4 & $20^{\text {th }}$ HRWYT $213 \times$ SUJATA & $-1.53^{* *}$ & $-0.66^{* *}$ & 0.05 & $-8.40^{* *}$ & $0.80^{* *}$ \\
\hline 5 & 20 $0^{\text {th }}$ HRWYT $235 \times$ GW 366 & $0.59^{* *}$ & $0.73^{* *}$ & 0.17 & $-3.43^{* *}$ & $3.50^{* *}$ \\
\hline 6 & $20^{\text {th }}$ HRWYT $235 \times$ SUJATA & $-0.78^{* *}$ & $1.45^{* *}$ & -0.03 & $-3.10^{* *}$ & $0.92^{* *}$ \\
\hline 7 & $45^{\text {th }}$ IBWSN $1021 \times$ GW 273 & $-1.52^{* *}$ & $1.79^{* *}$ & 0.02 & $-8.78^{* *}$ & $1.67^{* *}$ \\
\hline 8 & $45^{\text {th }}$ IBWSN $1021 \times$ RVW 4106 & $-0.30^{* *}$ & $0.65^{* *}$ & 0.08 & $-2.57^{* *}$ & $2.66^{* *}$ \\
\hline 9 & $14^{\text {th }}$ FHBSN6 $4188 \times \mathrm{GW} \times 366$ & $-1.25^{* *}$ & $0.74^{* *}$ & -0.03 & $-8.85^{* *}$ & $1.33^{* *}$ \\
\hline 10 & $14^{\text {th }}$ FHBSN6 $4188 \times$ SUJATA & $-0.19 * *$ & -0.03 & 0.06 & -0.32 & $1.08^{* *}$ \\
\hline 11 & $36^{\text {th }}$ SAWSN $3065 \times$ GW 366 & $-0.30^{* *}$ & $0.75^{* *}$ & $0.11^{*}$ & 0.7 & $2.14^{* *}$ \\
\hline 12 & $36^{\text {th }}$ SAWSN $3065 \times$ RVW 4106 & $-1.17^{* *}$ & $0.79 * *$ & 0.05 & $-5.76^{* *}$ & $1.13^{* *}$ \\
\hline 13 & PBW $658 \times$ SUJATA & $-1.58^{* *}$ & -0.48 & -0.06 & $-10.62^{* *}$ & $1.13^{* *}$ \\
\hline 14 & KB 2013-03 ×GW366 & $0.24^{* *}$ & $0.91^{* *}$ & 0.1 & 1.25 & $1.39^{* *}$ \\
\hline 15 & KB 2013-03 × SUJATA & -0.06 & $1.81^{* *}$ & $0.14^{* *}$ & 1.18 & $2.91^{* *}$ \\
\hline 16 & KB $2013 \times$ GW 273 & $-1.40^{* *}$ & 0.36 & $0.14^{* *}$ & $-4.61^{* *}$ & $1.15^{* *}$ \\
\hline 17 & KB $2013 \times$ RVW 4106 & 0.43 & 0.39 & 0.04 & 1.09 & $2.23^{* *}$ \\
\hline 18 & VW $921 \times$ RVW 4106 & $-1.44^{* *}$ & $1.43^{* *}$ & 0.09 & $-9.15^{* *}$ & $2.10^{* *}$ \\
\hline 19 & VW $921 \times$ SUJATA & $0.34^{* *}$ & 0.27 & 0.08 & -0.72 & 0.32 \\
\hline
\end{tabular}




\section{Agricultural Research \& Technology: Open Access Journal}

\begin{tabular}{|c|c|c|c|c|}
\hline SN & Crosses & $\begin{array}{l}\text { Canopy Temperature } \\
\text { Index }\end{array}$ & Biological Yield & Harvest Index \\
\hline & & SCA & SCA & SCA \\
\hline 1 & 33 ${ }^{\text {rd }}$ ESWYT $150 \times$ GW 366 & $0.02^{* *}$ & 0.14 & 1.19 \\
\hline 2 & 33 ${ }^{\text {rd }}$ ESWYT $150 \times$ RVW 4106 & -0.01 & 0.08 & $2.11^{* *}$ \\
\hline 3 & $20^{\text {th }}$ HRWYT $213 \times$ GW 273 & $0.03^{* *}$ & -0.4 & $4.58^{* *}$ \\
\hline 4 & $20^{\text {th }}$ HRWYT $213 \times$ SUJATA & 0.01 & 0.77 & -0.22 \\
\hline 5 & $20^{\text {th }}$ HRWYT $235 \times$ GW 366 & 0.01 & $1.10^{* *}$ & 1.95 \\
\hline 6 & $20^{\text {th }}$ HRWYT $235 \times$ SUJATA & -0.01 & $1.31^{* *}$ & -0.66 \\
\hline 7 & $45^{\text {th }}$ IBWSN $1021 \times$ GW 273 & -0.02 & $1.53^{* *}$ & 0.17 \\
\hline 8 & $45^{\text {th }}$ IBWSN $1021 \times$ RVW 4106 & 0 & $1.49^{* *}$ & 1.74 \\
\hline 9 & $14^{\text {th }}$ FHBSN6 $4188 \times \mathrm{GW} \times 366$ & $0.03^{* *}$ & 0.6 & 0.66 \\
\hline 10 & $14^{\text {th }}$ FHBSN $64188 \times$ SUJATA & $-0.04 * *$ & $1.97^{* *}$ & -1.06 \\
\hline 11 & $36^{\text {th }}$ SAWSN $3065 \times$ GW 366 & $-0.02^{* *}$ & -0.01 & $3.25^{* *}$ \\
\hline 12 & $36^{\text {th }}$ SAWSN $3065 \times$ RVW 4106 & 0 & 0.54 & $-2.55^{* *}$ \\
\hline 13 & PBW $658 \times$ SUJATA & -0.01 & -0.07 & 0.94 \\
\hline 14 & KB 2013-03 ×GW366 & 0 & $0.80^{*}$ & 0.5 \\
\hline 15 & KB 2013-03 × SUJATA & 0 & $-2.57^{* *}$ & $6.44^{* *}$ \\
\hline 0.16 & KB $2013 \times$ GW 273 & 0 & $-0.82 * *$ & 1.93 \\
\hline 17 & KB $2013 \times$ RVW 4106 & $0.02^{* *}$ & 0.76 & 1.78 \\
\hline 18 & VW $921 \times$ RVW 4106 & $-0.03 * *$ & -0.75 & $3.14^{* *}$ \\
\hline 19 & VW $921 \times$ SUJATA & 0 & $1.48^{* *}$ & -1.17 \\
\hline
\end{tabular}

\section{Heterosis}

The aim of estimation of heterosis in the present study has to spot out the best combination of parents giving high degree of useful heterosis and characterization of parents for their genotypic worth for future use in breeding programme. All characters had shown considerable amount of heterosis over better parent (Heterobeltiosis) and mid parent (relative heterosis). Present finding are in confirmation with Devi et al. [10]. The degree of heterosis however differed for different

characters. Data presented in Table 5 revealed that 4 cross viz., 'KB2013×RVW4106', '33rd ESWYT150×RVW4106', 'KB2013$03 \times$ SUJATA' and 'KB2013-03×RVW4106' recorded significant heterobeltiosis and relative heterosis in positive direction for grain yield, while another 3 cross '33rd ESWYT150×GW366', '45th IBWSN1021×RVW4106' and 'PBW658×RVW4106' recorded significant positive relative heterosis for grain yield. Devi et al. [10] also reported similar result [11-15].

Table 5: Heterosis over better parent and mid parent for grain yield and its attributes.

\begin{tabular}{|c|c|c|c|c|c|c|c|c|c|c|c|c|c|c|}
\hline \multirow[t]{2}{*}{ Crosses } & \multicolumn{2}{|c|}{ Grain Yield/Plant } & \multicolumn{2}{|c|}{$\begin{array}{l}\text { Days to } \\
\text { Maturity }\end{array}$} & \multirow[b]{2}{*}{ BP } & \multirow[b]{2}{*}{ MP } & \multicolumn{2}{|c|}{ Plant Height } & \multicolumn{2}{|c|}{ Tillers/Plant } & \multicolumn{2}{|c|}{$\begin{array}{l}\text { 1st Inter-Node } \\
\text { Length }\end{array}$} & \multicolumn{2}{|c|}{ Spike Length } \\
\hline & BP & MP & BP & MP & & & BP & MP & BP & MP & BP & MP & BP & MP \\
\hline $\begin{array}{l}\text { KB2013× } \\
\text { RVW4106 }\end{array}$ & $16.33^{* *}$ & $16.88^{* *}$ & $-2.84^{* *}$ & -1.44 & $-1.09 * *$ & $-2.61^{* *}$ & $-2.59 * *$ & $-10.14^{* *}$ & -9.49 & $-6.87^{* *}$ & $-12.78^{* *}$ & $-9.60^{* *}$ & -1.09 & $9.43^{* *}$ \\
\hline $\begin{array}{c}33^{\text {rd }} \\
\text { ESWYT150 } \\
\times \text { RVW4106 }\end{array}$ & $11.24^{* *}$ & $16.05^{* *}$ & -1.15 & -0.29 & $-2.52^{* *}$ & $-1.81^{* *}$ & 0.05 & $4.28^{* *}$ & $15.28^{* *}$ & $18.57^{* *}$ & 0 & $3.25^{* *}$ & $-22.19 * *$ & $-19.35^{* *}$ \\
\hline $\begin{array}{l}\text { KB2013-03 } \\
\times \text { SUJATA }\end{array}$ & $3.90^{* *}$ & $7.67^{* *}$ & $-2.31^{* *}$ & 0 & $-1.08^{* *}$ & -0.91 & $-4.78^{* *}$ & $-2.33^{* *}$ & $-21.69^{* *}$ & $-14.47^{* *}$ & 1.38 & $7.37^{* *}$ & $-9.98^{* *}$ & 0.59 \\
\hline $\begin{array}{l}\text { KB2013-03 } \\
\times \text { RVW4106 }\end{array}$ & $3.20^{* *}$ & $7.28^{* *}$ & $-3.91^{* *}$ & -1.71 & $1.46^{* *}$ & $1.65^{* *}$ & $-15.69 * *$ & $-15.48^{* *}$ & 1.45 & 2.19 & -0.99 & 2.22 & $-25.53^{* *}$ & $-17.16^{* *}$ \\
\hline $\begin{array}{c}33^{\text {rd }} \\
\text { ESWYT150 } \\
\times \text { GW366' }\end{array}$ & 0.34 & $4.99 * *$ & $-2.30^{* *}$ & -1.45 & $-2.88^{* *}$ & $-1.82^{* *}$ & $-3.43^{* *}$ & 0.19 & -1.19 & 6.41 & -2.24 & -0.49 & $-5.37^{* *}$ & 2.07 \\
\hline $\begin{array}{c}45^{\text {th }} \\
\text { IBWSN1021 } \\
\times \text { RVW4106 }\end{array}$ & -0.53 & $2.90 * *$ & $-6.04^{* *}$ & $-3.12^{* *}$ & -0.73 & -0.55 & $-3.53^{* *}$ & $-3.33^{* *}$ & $-10.39 * *$ & -4.83 & $-10.07^{* *}$ & $-7.62^{* *}$ & $-9.63^{* *}$ & $-9.30 * *$ \\
\hline
\end{tabular}




\section{Agricultural Research \& Technology: Open Access Journal}

\begin{tabular}{|c|c|c|c|c|c|c|c|c|c|c|c|c|c|c|}
\hline $\begin{array}{l}\text { PBW658 × } \\
\text { RVW4106 }\end{array}$ & $2.43^{* *}$ & $2.47^{* *}$ & -1.16 & -0.58 & $-2.53^{* *}$ & $-2.00^{* *}$ & $-4.36^{* *}$ & $-2.41^{* *}$ & -7.14 & -5.8 & -1.38 & -0.17 & $11.14^{* *}$ & $-7.30^{* *}$ \\
\hline & \multicolumn{2}{|c|}{$\begin{array}{l}\text { Weight of Spike/ } \\
\text { Plant }\end{array}$} & \multicolumn{2}{|c|}{$\begin{array}{l}\text { Weight of } \\
\text { Grain/Spike }\end{array}$} & $\begin{array}{l}\text { Grain/ } \\
\text { Spike }\end{array}$ & \multirow[b]{2}{*}{ MP } & \multirow{2}{*}{$\begin{array}{c}\begin{array}{c}\text { Test } \\
\text { Weight }\end{array} \\
\text { BP } \\
\end{array}$} & \multirow[b]{2}{*}{ MP } & \multicolumn{2}{|c|}{$\begin{array}{c}\text { Canopy } \\
\text { Temperature Index }\end{array}$} & \multicolumn{2}{|c|}{ Biological Yield } & \multicolumn{2}{|c|}{ Harvest Index } \\
\hline Crosses & BP & MP & BP & MP & BP & & & & BP & MP & BP & MP & BP & MP \\
\hline $\begin{array}{l}\text { KB2013 × } \\
\text { RVW4106 }\end{array}$ & $4.31^{* *}$ & $8.82^{* *}$ & 4.21 & 5.65 & -7.73 & $-6.84^{* *}$ & $12.54^{* *}$ & $13.52^{* *}$ & $-21.92^{* *}$ & $-19.72^{* *}$ & 1.55 & 7.49 & 2.4 & 3.59 \\
\hline $\begin{array}{l}\text { 33rd ESWYT150 } \\
\times \text { RVW4106 }\end{array}$ & $11.80^{* *}$ & $13.55^{* *}$ & 6.27 & 7.07 & $-17.06^{* *}$ & -12.08 & $10.44^{* *}$ & $14.33^{* *}$ & $-21.13^{* *}$ & -20 & $4.00^{* *}$ & $12.00^{* *}$ & -0.08 & 3.3 \\
\hline $\begin{array}{l}\text { KB2013-03 } \\
\text { SUJATA }\end{array}$ & 3.36 & $9.76^{* *}$ & 4.96 & 6.1 & -5.51 & $7.04^{* *}$ & $3.67^{* *}$ & $7.18^{* *}$ & $-16.90^{* *}$ & $-16.08^{* *}$ & 0.03 & 2.67 & -1.53 & $4.64^{* *}$ \\
\hline $\begin{array}{l}\text { KB2013-03 × } \\
\text { RVW4106 }\end{array}$ & 1.28 & 3.4 & -0.97 & 1.49 & -18.22 & $-8.63^{* *}$ & $5.46^{* *}$ & $8.78^{* *}$ & $-18.06^{* *}$ & $-16.31^{* *}$ & $8.39 * *$ & $10.78^{* *}$ & -4.81 & -3.16 \\
\hline $\begin{array}{l}33^{\text {rd }} \text { ESWYT150 } \\
\times \text { GW366 }\end{array}$ & -1.13 & $5.47^{* *}$ & -3.95 & 2.46 & -3.23 & -2.2 & 0.54 & $4.96^{* *}$ & $-21.79 * *$ & $-18.12^{* *}$ & $5.29 * *$ & $14.44^{* *}$ & $-19.86^{* *}$ & $-9.28^{* *}$ \\
\hline $\begin{array}{l}4^{\text {th }} \text { IBWSN1021 } \\
\times \text { RVW4106 }\end{array}$ & -1.07 & 0.5 & 0.76 & 0.76 & $-10.13^{* *}$ & -3.97 & -0.55 & 1.8 & $-14.29^{* *}$ & $-13.67^{* *}$ & $8.73^{* *}$ & $13.95^{* *}$ & $-11.04^{* *}$ & $-9.82^{* *}$ \\
\hline $\begin{array}{l}\text { PBW658 } \times \\
\text { RVW4106 }\end{array}$ & $-4.04^{* *}$ & -0.85 & -1.18 & 2.45 & -3.21 & 2.63 & -4.33 & 0.23 & $-26.09 * *$ & $-26.09 * *$ & 2.03 & $4.95^{* *}$ & $-5.51^{*}$ & -2.91 \\
\hline
\end{tabular}

Cross 'KB2013×RVW4106' was also reported significant positive heterobeltiosis and relative heterosis for weight of spike/plant and test weight. Cross '33rd ESWYT150×RVW4106' also showed significant heterobeltiosis and relative heterosis in positive direction for tillers/plant, weight of spike/plant, test weight and biological yield. Cross 'KB2013-03×RVW4106' was also reported significant positive heterobeltiosis and relative heterosis for days to maturity, test weight and biological yield [16-18]. Cross 'KB2013-03×SUJATA' showed significant positive relative heterosis for 1 st inter-node length, weight of spike/ plant, test weight, biological yield and harvest index, while significant positive heterobeltiosis was reported for test weight and canopy temperature index. Cross '33rd ESWYT150×GW366' was also reported significant positive relative heterosis for weight of spike/plant, test weight, and biological yield. Cross '45th IBWSN1021×RVW4106' was also reported significant positive heterobeltiosis and relative heterosis for biological yield. Cross 'PBW658×RVW4106' also showed significant positive relative heterosis for biological yield. Further it was noticed that both significant heterobeltiosis or/and relative heterosis were recorded for attributing traits without showing heterosis for grain yield [19-23].

\section{Conclusion}

Genotypes 33 ${ }^{\text {rd }}$ ESWYT150, PBW658, KB2013-03, KB2013 and VW921, GW273 and SUJATA were identified good general combiner for grain yield per plant and its components in wheat [24-27]. Cross 33 ${ }^{\text {rd }}$ ESWYT 150 X GW 366, 33 ${ }^{\text {rd }}$ ESWYT $150 \times$ RVW4106, 20 $0^{\text {th }}$ HRWYT $213 \times$ GW273, 20 $0^{\text {th }}$ HRWYT $213 X$ SUJATA, 20 th $H R W Y T$ 235XGW366, 20 $0^{\text {th }}$ HRWYT 235 $X$ SUJATA, $45^{\text {th }}$ IBWSN1021 $X$ GW273, 45 ${ }^{\text {th }}$ IBWSN1021 $X$ RVW4106, $14^{\text {th }}$ FHBSN6418XGW366, $14^{\text {th }}$ FHBSN6418 X SUJATA, $36^{\text {th }}$ SAWSN3065 X GW366, 36 th $^{\text {th }}$ SAWSN3065XRVW4106,
PBW658XSUJATA, KB2013-03 X GW366, KB2013-03 X SUJATA, KB2013XGW273, KB2013XRVW4106, VW921XGW366, VW921 X SUJATA were identified good specific combiner for grain yield per plant and its components in wheat so it may be suitable for exploitation in future plant breeding programme [28-30].

Additive variance played the major role in determining all of the characters. Some of the cross recorded significant heterosis for grain yield, thereby, $[31,32]$ indicating further scope of hybrid development in near future. The crosses KB2013-03XSUJATA was desirable for selecting higher yielding and short duration plant, while cross KB2013XRVW4106 was desirable for the selection of high yielding dwarf plant over the better parent. The crosses KB2013-03XRVW4106, KB2013XSUJATA and KB201303XSUJATA were suitable for selection of high yielding and short duration plant over the mid parent. The cross ESWYT $150 \mathrm{X}$ RVW4106 was desirable for selecting higher yielding and short duration plant over both better and mid parent [33].

\section{Suggetions for Further Work}

The following relevant suggestions could be drawn for further scope of improvement from the present study: Good general combiners like 33 rd ESWYT150, PBW658, KB2013-03, KB2013 and VW921, GW273 and SUJATA could be used in the development of high yielding varieties through the simple/recurrent selection from promising segregating cross generations in wheat. New recombinant promising single plants may be selected in F2 generations of good cross 33 ${ }^{\text {rd }}$ ESWYT150XRVW4106, KB201303XSUJATA and 33 ${ }^{\text {rd }}$ ESWYT150XGW 366 involving one of the good specific combining parents. The cross $33^{\text {rd }}$ ESWYT 150XRVW4106 was found higher yielding and short duration plant over both better and mid parent. Thus it was very desirable for further breeding programme while selecting the genotype $[34,35]$. 


\section{References}

1. Kempthrone O (1957) An introduction to genetical statistics. John Wiley and Sons, New York, USA.

2. Singh K, Singh UB, Sharma SN (2013) Combining ability analysis for yield and its components in bread wheat (Triticum aestivum L.). J Wheat Res 5(1): 63-67.

3. Kapoor E, Mondal S, Dey T (2011) Combining ability analysis for yield and yield contributing traits in winter and spring wheat combinations. J Wheat Res 3(1): 52-58.

4. Kumar A, Mishra VK, Vyas RP, Singh V (2011) Heterosis and combining ability analysis in bread wheat (Triticum aestivum L.). J PL Breeding and Crop Sci 3(10): 209-217.

5. Kandil AA, Sharief AE, Hasnaa SM, Gomaa (2016) Estimation of general and specific combining ability in bread wheat (Triticum aestivum L.). Int J Agri R 8(2): 37-44.

6. Khiabani BN, Aharizad S, Mohammadi SA (2015) Genetic analysis of grain yield and plant height in full diallel crosses of bread wheat. Biological Forum - An Int J 7(1): 1164-1172.

7. Kalhoro FA, Rajpar AA, Kalhoro SA, Mahar A, Ali A, et al. (2015) Heterosis and combing ability in F1 population of hexaploid wheat (Triticum Aestivum L.). American J PL Sci 6(7): 1011-1026.

8. Aslam R, Munawar M, Salam A (2014) Genetic architecture of yield components accessed through line $\times$ tester analysis in wheat (Triticum aestivum L.). Universal J PL Sci 2(5): 93-96.

9. Raj P, Kandalkar VS (2013) Combining ability and heterosis analysis for grain yield and its components in wheat. J Wheat Res 5(1): 45-49.

10. Devi L, Swati GP, Singh M, Jaiswal JP (2013) Heterosis studies for yield and yield contributing traits in bread wheat (Triticum aestivum L.). The Bioscan 8(3): 905-909.

11. Ajmal S, Asif M, Munir M (2004) Implication of combining ability: Analysis of some characteristics of spring wheat. Quarterly Sci Vision 9(1-2): 1-5.

12. Akbar M, Anwar J, Hussain M, Qureshi MH, khan S (2009) Line $\times$ tester Analysis in bread wheat (Triticum aestivum L.). J Agric Res 47(1): 411.

13. Akram Z, Ajmal SU, Khan KS, Qureshi R, Zubair R (2004) Combining ability estimates of some yield and quality related traits in spring wheat (Triticum aestivum L.). Pak J Bot 43(1): 221-231.

14. Anonymous (2015) Progress Report of All India Coordinated Wheat and Barley Improvement Project, 2014-2015, IIW and BR, India, pp. 1: 1 .

15. Ashadusjman M, shamsuddoha M, Alam MJ, Begum MO (2012) Combining ability and gene action for different root characters in spiring wheat. J Env Sci and natural resources 5(2): 73-76.

16. Awan SI, Malik MF, Siddique M (2005) Combining ability analysis in inter-varietal crosses for component traits in hexaploid wheat. J Agric and social Sci 1(4): 316-317.

17. Batool A, Noorka LR, Afzal M, Syed AH (2013) Estimation of heterosis, heterobeltiosis and potence ratio over environments among pre and post green revolution spring wheat in pakistan. J Basic and Applied Sci 9: 36-43.

18. Cifci EA (2012) Estimate of heterosis, correlation and path analysis for grain yield per spike and some agronomic traits on durum wheat (Triticum durum). The J of Animal and PL Sci 22(3): 747-752.
19. Desale CS, Mehta DR, Singh AP (2014) Combining ability analysis in bread wheat. J of Wheat Res 6(1): 25-28.

20. Ilkar E, Tonk FA, Tosun M (2010) Heterosis for yields and its components in bread wheat crosses among powdery mildew resistant and susceptible genotype. Pak J Bot 42(1): 513-522.

21. Hussaim M, Irshadi A, Ammari A, Mahmood K, Aziz MK, et al. (2014) Heterotic studies in spring wheat across two environments. J Bio and Env Sci 5(5): 143-148.

22. Ismail KAS (2015) Heterosis and combining ability analysis for yield and its Components in Bread Wheat (Triticum aestivum L.). Int J Curr Microbiol App Sci 4(8): 1-9.

23. Istipliler D, Ilker E, Tonk FA, Civi G, Tosun M (2015) Line $\times$ tester analysis and estimating combining abilities for yield and some yield components in bread wheat. Turkish J Field Crops 20(1): 72-77.

24. Jain S, Sastry EVD (2012) Heterosis and combining ability for grain yield and its contributing traits in bread wheat (Triticum aestivum $L$.). J Agric and Allied Sci 1:1.

25. Jatav M, Jatav SK, Kandalkar VS (2014) Combining ability and heterosis analysis of morpho- physiological characters in wheat. Ann PL and Soil Res 16(2): 79-83.

26. Khan MA, Ahmad N, Akbar M, Rehman AU, Iqbal MM (2007) Combining ability analysis in wheat. Pak J Agri Sci 44(1): 2-5.

27. Khatun MT, Bari MAA, Zaman MA, Begum H, Akter S (2010) Hetrosis estimates in F2 diallel population of spring wheat at two different cultural conditions. Bangladesh J Agric Res 35(3): 413-422.

28. Lohithaswa HC, Desai SA, Hanchinal RR, Patil BN, Math KK, et al. (2013) Combining ability in tetraploid wheat for yield, yield attributing traits, quality and rust resistance over environments. Karnataka J Agric Sci 26(2): 190-193.

29. Nour A, Nadya AR, Fateh H, Mostafa A (2011) Line $\times$ tester Analysis for yield and its traits in bread wheat. Egypt J Agric Res 89(3).

30. Padhar PR, Chovatia VP, Jivani LL, Dobariya KL (2013) Combining ability analysis over environments in diallel crosses in bread wheat (Triticum aestivum L.). Internat J agric Sci 9(1): 49-53.

31. Pawar KK Yadav SK, Baghel KS, Singh AK (2014) Study of diallel analysis in bread wheat for yield and its component. Int J scientific Res 3(3).

32. Saeed A, Chowdhary MA, Khaliq N, Johar MZ (2001) Line x tester analysis for some morpho-physiological traits in bread wheat. Int J Agric and Bio 3(4): 444-447.

33. Sharma I, Shoran J, Singh G, Tyagi BS (2011) Wheat Improvement in India. Souvenir of $50^{\text {th }}$ All India Wheat and Barley Res Workers Meet, USA, p. 11.

34. Singh H, Sharma SN, Sain RS (2004) Heterosis studies for yield and its components in bread wheat over environments. Hereditas 141(2): 106-114.

35. Zeeshan M, Arshad W, Ali S, Tariq M, Hussain M, et al. (2013) Estimation of combining ability effects for some yield related metric traits in intraspecific crosses among different spring wheat (Triticum aestivum L.) genotypes. International J Advanced Res 1(3): 6-10. 
This work is licensed under Creative Commons Attribution 4.0 License

DOI:10.19080/ARTOAJ.2017.13.555868
Your next submission with Juniper Publishers will reach you the below assets

- Quality Editorial service

- Swift Peer Review

- Reprints availability

- E-prints Service

- Manuscript Podcast for convenient understanding

- Global attainment for your research

- Manuscript accessibility in different formats ( Pdf, E-pub, Full Text, Audio)

- Unceasing customer service

Track the below URL for one-step submission https://juniperpublishers.com/online-submission.php 